\title{
Colorectal Juvenile Polyp
}

National Cancer Institute

\section{Source}

National Cancer Institute. Colorectal Juvenile Polyp. NCI Thesaurus. Code C5681.

A non-neoplastic hamartomatous polyp that arises from the colon and rectum. It is characterized by the presence of tortuous and cystically dilated glands, edematous changes, and inflammation. 